\title{
An Event-driven Cooperation Model of Information Appliances in the Intelligent Home
}

\author{
Hua Peng \\ College of Information Science and Engineering \\ JiShou University \\ JiShou, China,15074339004 \\ penghua_47@163.com
}

\begin{abstract}
In the current system of the intelligent home, it control information appliances with the sole mode, can not realize the cooperation between of them, its intelligence can not get sufficient play. Based on the principle that the cooperation of information appliances are often event-driven, in this paper, the concept of event is proposed. The architecture model of the intelligent home's system based on event-driven and expert-system is studied by the method of object-oriented analysis and design. The process of system, which is composed with event capture, event multicast and event response is given. The experimental results show that the system model is feasible. The research of this paper has important application value.
\end{abstract}

Keywords-Event-driven; Equipment's cooperation; Information appliance; Object-oriented

\section{INTRODUCTION}

With the development of the economic level and the social science and technology, people request more for the intelligence and humanization of the intelligent home. In the current system of the intelligent home, although it has accomplish data transferring of three kinds of devices, house security monitoring, and other functions, the automation of information appliances has more enhancements than before ${ }^{[1]}$; However, all kinds of operations of information appliances are only simple "mechanization" mode, all sorts of equipments of information appliances (hereafter referred to as equipments) can't call each other and work together on the service, the intelligence is not enough and shall be further improved. People expect that the future system of the intelligent home can drive the information appliances to react automatically according to the change of environment, make their living environment more comfortable, intelligent and modern.

This paper proposed a architecture model of the intelligent home based on the technology of the event-driven and expert system, by capturing events and reasoning rules, make it's process automated that events drive the equipments to cooperate in the intelligent home.

\section{EVENTS OF INFORMATION APPLIANCE}

The interconnection network of the intelligent appliances in the intelligent home constitute a specially distributed computing environment, information appliances can cooperation only if they can identify each other. Therefore, we need a unified, abstract language to define the equipments of the information appliances and describe their functions, make all information appliances are built on the same language platform. This paper use the IAIDL (Information Appliance Interface Definition Language) to define and describe the equipments of the information appliances, its content includes: the equipment's attributes, operations, and events. About IAIDL details can see reference [2].

In the intelligent home's network, it will trigger an event when a state or attribute's data changes, which belong to a equipment of information appliance. An event can be an open operation or a temperature change of an air-conditioning, it constitutes the basic information unit which interactive between the equipment of information appliance and environment.

In the intelligent home's network, an event can be regarded as the trigger action which the attributes of an equipment get for a state. Events are generated by running of the equipments, at the same time, they relate to the following factors: (1) association member: the equipment which hope its events are activated to execute an operation; (2) event producer: the equipment which activate event by its activities; (3) event sensor: the module which is responsible for monitoring events and transfer event information to the event sender; (4) event sender: the module which multicast events to all the association members; (5) Agent : the module which response events and drive association members to finish the corresponding operation.

\section{ARCHITECTURE}

\section{A. The Architecture Model}

The model of system's architecture is shown in Figure 1., In the intelligent home, each equipment connect with home network by a agent. Each equipment can be event producer which sends its events to home network, and can be association member which receive some events from home network. As home gateway is the main centre of the whole system, its embedded database stores all information, including each equipment's registration information (network address, attributes, operations, events) and cooperation rules; The event monitoring service program manage and monitor the events in the system; Its E-C (expert-control) cooperation control system is used for managing and reasoning the cooperation rules of 
equipments, and passing the rules and reasoning result to the corresponding equipment's agent. Client control module can be a PC or a versatile controller of information appliance(about the details of the versatile controller of information appliance can see reference[3]), using its GUI interface, it can get all of information of each equipment from the home gateway in the system, and can customize the cooperation rules among the equipments, and will submit the rules to the home gateway, and E-C cooperation control system is responsible for generating and storing the rules. Also client control module can directly access the information of equipment or directly control ${ }^{[3]}$.

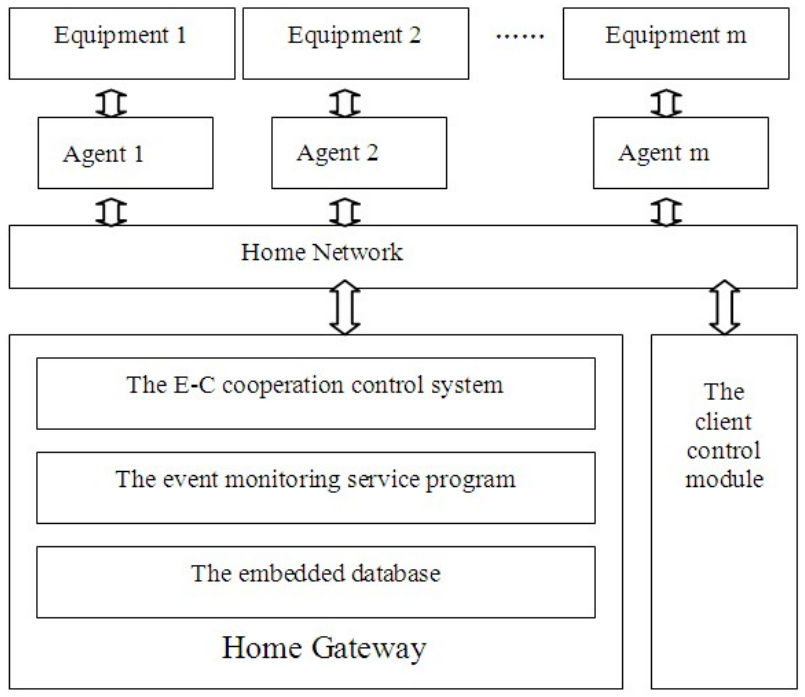

Figure 1 The model of system's architecture

\section{B. Agent Of equipment}

The equipment's agent is shown in Figure 2. An Agent is consist of the equipment interface module, data conversion module, rule storage module, rule conversion module and communication module. The equipment interface module implement that interact with the external equipment, get the event information which it produced before, access the data of its attributes, and send commands to drive it to perform the appointed action; The Data conversion module will convert the information of data, which get from the equipment interface module, to a standard data format, or will convert a standard data information, which get from the communication module, to the specific data format that the equipment can identify ${ }^{[4]}$. The rule storage module will store all the rules and reasoning results for the rule conversion module which the E-C cooperation control system pass them to the agent; the rules conversion module will receive the equipment's event from home network and matching the rules or reasoning results that has stored in the rules storage module, and pass the equipment operation driven by the event to the equipment by the equipment interface module, and drive it to execute the corresponding operation. The communication module interact with home network environment, it will send the agent's corresponding event and data information to home network in the form of message package, and receive the message package from home network.
Additionally, when the client control module directly send an operation command to an equipment, the corresponding agent will get some message packages from home network; after the agent unpack this packages, it will pass directly the operation command to the equipment by the equipment interface module and drive it to perform the appointed action.

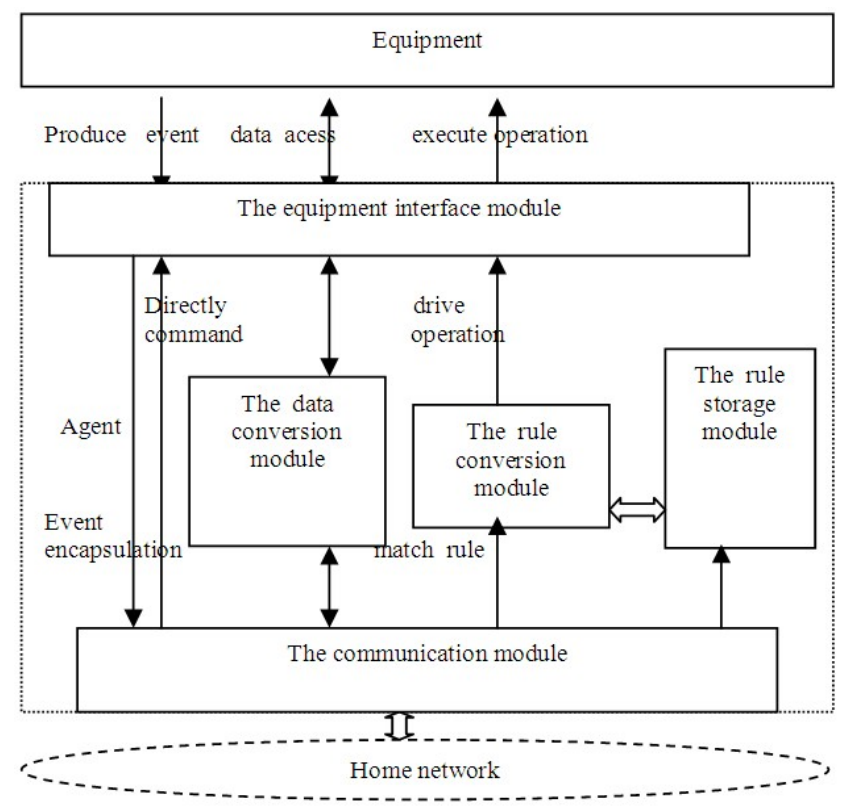

Figure 2 The equipment's agent

\section{The System Procedure Based On Event-driven}

The system procedure based on event-driven includes three main parts: event capture, event multicast and event result confirmed. In home network, an event producer produced an event, the event monitoring service program will capture it, and sort it, thereby decide the sequence of multicasting events. By using the multicast technology, events are sent to all the association members ${ }^{[5]}$, each association member will respond the events, and executes the corresponding operations, finally will return the response results to the event monitoring service program, which will confirm that all the association members have responded the events normally. Figure 3 describes the whole procedure of event capture, multicast, respond and process in the system of intelligent home. The procedure describes as follows:

- According to the change of information appliance 1's running status, it will produce an event, and the corresponding agent 1 will capture the event.

- Agent 1 will encapsulate the event, generate a message package and send it to home network.

- After the event monitoring service program in the home gateway has monitor the event messages by the event sensor, it will analysis and sort of events (data structure is priority queue, the event which has high priority will be processed preferentially, and if two event in the queue has the same priority, the strategy of FIFO is used), and then multicast them to every association member in home network environment. 
- After agent 2 have got the message packages, it will unpack this packages, then match the rules or reasoning results that are stored in the rule storage module, and pass the equipment operation information, which is driven by the events, to information appliance 2;

- After information appliance 2 has received the equipment operation information, it will execute the corresponding operation. When it finish the operation, it will send the result of execution to the event monitoring service program in the form of the message packages similarly;

- The event monitoring service program will capture the event message packages, and confirm the equipments have accomplish the cooperation.

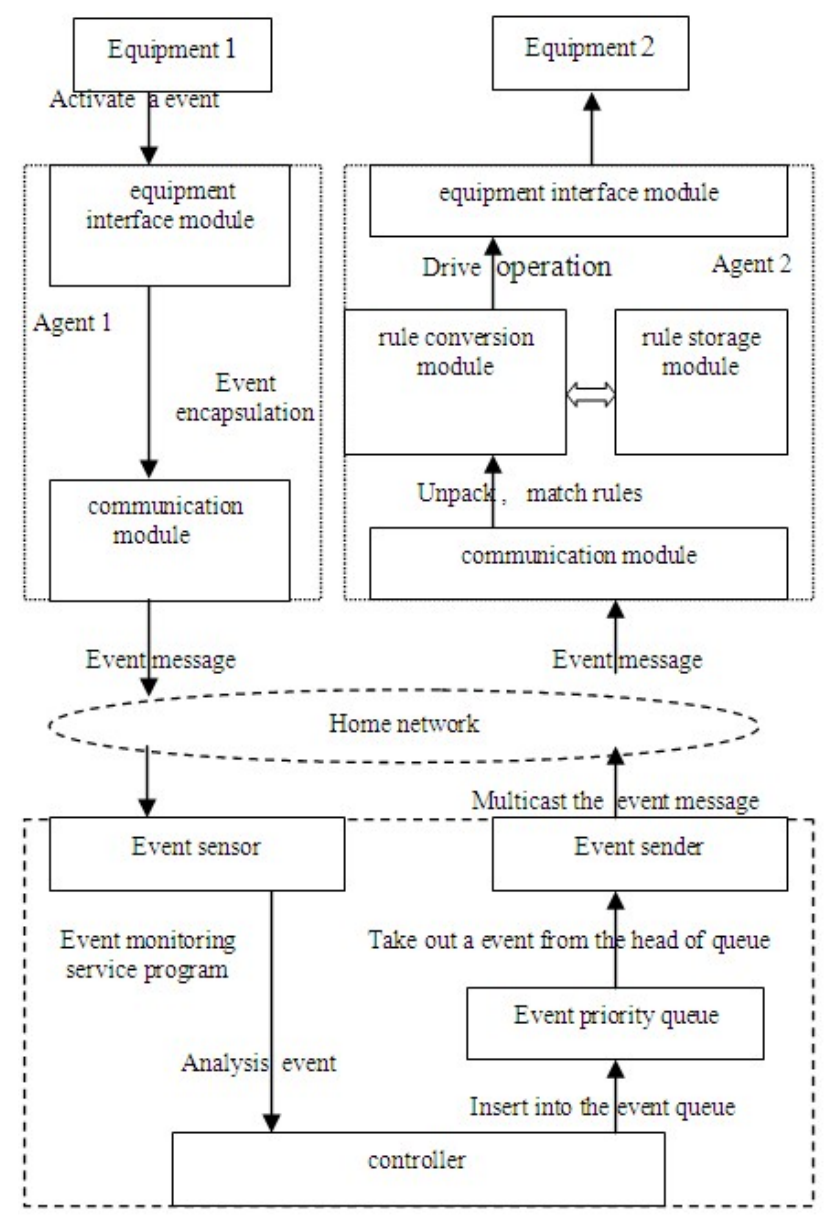

Figure 3 The procedure of the system based on event-driven

\section{SYSTEM SIMULATION AND ANALYSIS}

According to the model of the system, this paper has simulated on the personal computers (PCs). In the simulation environment, three program, which develop in Delphi7, are simulated home gateway, network air conditioning, network television (hereinafter referred to as the PHG, PAC and PTV) run on the different PC, the PCs are connected in network; And the "turn on the network television---> turn on the network air conditioning "cooperation rule has been established, so the network air conditioning becomes the" turn on the network television " event's association member, and the rule also be stored in the rule storage module in PAC. Used between of them the event message package's format is as follows:

TABLE 1. FORMAT OF THE EVENT MESSAGE PACKAGE

\begin{tabular}{|l|l|l|l|c|}
\hline Equipment ID & Event ID & Send Time & Result ID & Priority \\
\hline
\end{tabular}

Thereinto, equipment ID is the ID of information appliance, event ID is the number of the event supported by the equipment,( equipment ID and event ID are defined by system automatically and uniquely), send time is the time of generating the event message package by the equipment, result ID is used for judging the current event message package is or not the confirm information of the implement result of some event before, and if so, it would be equal to an event ID before, otherwise, it is empty; Priority is one value of predefined 0-6 priority level by the system (when a equipment was registered in the system, the system or administrator would have defined every event's priority in the equipment).

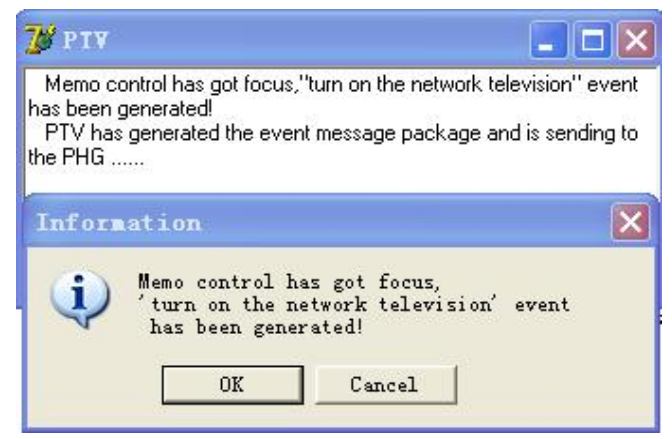

Figure 4 PTV’s interface

As shown in figure 4, 5, 6, the memo control in every program will record the program's actions. When the mouse clicked in the memo control (an equipment) on PTV (the memo control got focus, and its state has changed, so simulated that PTV generated a "turn on the network television" event), the PTV (the agent) would generate an event message package and sent to the PHG. After receiving the event message package from the PTV, the PHG would analyse the package; for example, check whether "result ID" is empty, if is empty, need multicast the event to all the association members in the system at the right time, if is not, needn't to multicast; check the value of "priority" to decide the position of the event queue into which the event would be inserted, the PHG would take out the event on the head of the event queue according to the priority level from high to low order, and further processed. To ensure that all events have the opportunity to process, therefore, set a time threshold (such as 3 seconds), the lower priority event had not been processed when exceeding the time threshold value, would be improved its priority automatically, avoid it is in a state of permanent hungry. After received the " turn on the network television " event message package from the PHG, the PAC would search the corresponding rule in its internal rule storage module, and found the " turn on the network television--> turn on the network air conditioning " cooperation rule, then would call the internal function produce to create an " 
open the network air conditioning "dialog control. Finally, the PAC would generate a confirm event message package to sent to the PHG, we can see the record in PHG's memo control (as figure 5). So this paper has simulated the cooperation of the equipments (between the network television and the network air conditioning) based on event-driven technology.

THS PHG
Received a event message package from the PTV!
Analysis the event message package.....
The content of the event message package is:
Equipment ID: 1
Event ID: 1
Send time: $14: 27: 32: 953$
Result ID: NULL
Priority: 6
Insert the event message package into the event queue...
The event's position of queue is: 1
Take out the event which is at head of the event queue.
Find the association member of the event is: PAC
Multicast the event message package to its association
members......
Received a event message package from the PAC!
Analysis the event message package......
The content of the event message package is:
Equipment ID: 2
Event ID: 11
Send time: $14: 27: 33: 446$
Result ID: 1
Priority: 1

Figure 5 PHG's interface

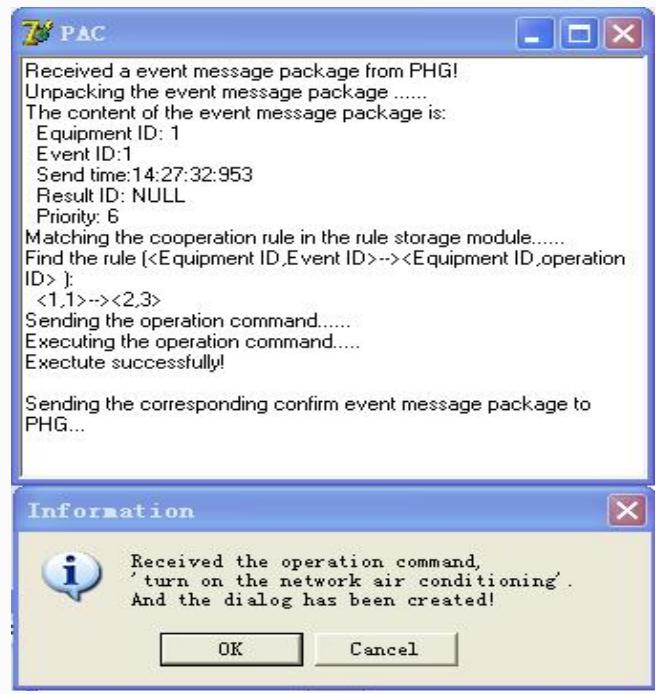

Figure 6 PAC’s interface

The model of the system uses response time as the evaluation index of cooperation performance, the response time is defined as: response time is equal to subtract some action's finish time caused by a event from the time of generating the event, in the above example, subtract the time of generating the confirm event message package where come from PAC from the time of generating "turn on the network television" event message package in PTV equal to the response time of the cooperation which is between the PAC and the PTV. This paper assumes that in any intelligent home environment, the number of the equipment of information appliances has not exceed 30, each equipment has 10 events and stores 10 cooperation rules. As shown in figure 7, with the number of equipment increasing, the system's response time is linear growth, but in the extreme situation (equipment's number equals to 30 ), the system response time is 12.8 seconds. The experimental results show that the event-driven cooperation model of information appliances is feasible.

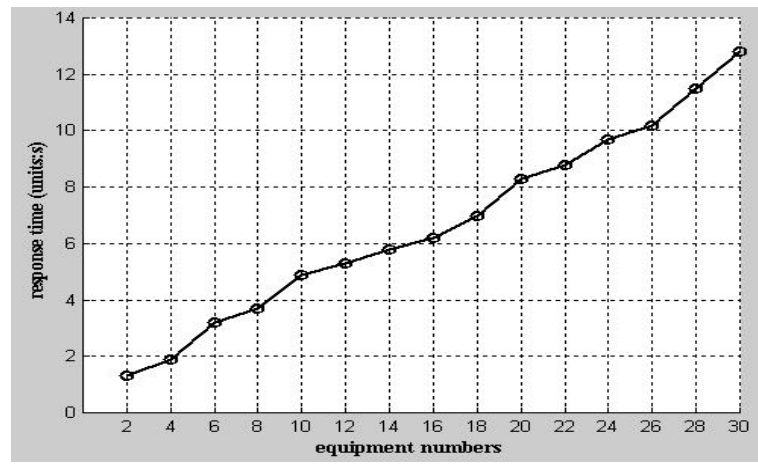

Figure 7 The change of response time

\section{CONCLUSIONS}

With the rapid development of the information technology, network technology and object-oriented technology, they provide a broad prospect for developing the information appliances and intelligent home. Based on the principle that the cooperation of information appliances are often event-driven, in this paper, the concept of event is proposed. The architecture model of the intelligent home's system based on event-driven and expert-system is studied by the method of object-oriented analysis and design. The process of system, which is composed with event capture, event multicast and event response is given. Finally, the model of the system is verified by simulation experiment. By introducing the event-driven mechanism to the cooperation of information appliances, it has improved the intelligence and convenience of information appliances and intelligent home.

\section{REFERENCES}

[1] Cong Zhu, Hong Liu, Hua Peng and Zanyu Tang, "Researh and design of smart home by using expert system” ,Microcomputer information, vol. 8, pp. 73-74, 2008.

[2] Huihua Huang, "The design and implementation of the remote monitoring system based on the information home appliances interface definition language", Changsha: Hunan normal university, 2005.

[3] Hua Peng, "The research and design of the versatile controller of information appliance in the intelligent house”, Changsha: Hunan normal university, 2008.

[4] Xiangdong Chen, "New system based on event-driven and service-oriented business activity monitoring design and implementation",Application research of computers, vol. 29(3), pp. 977-980, 2012.

[5] Lin Yuan, Xinjia Zhang and Fei Li, "Research of heterogeneous data exchange system based on eventdriven mechanism" ,Computer technology and development, vol. 21(12), pp. 100-104, 2011. 